\title{
High-power laser light source for near-field optics and its application to high-density optical data storage
}

Afshin Partovi, David Peale, Matthias Wuttig, Cherry A. Murray, George Zydzik, Leslie Hopkins, Kirk Baldwin, William S. Hobson, James Wynn, John Lopata, Lisa Dhar, Rob Chichester, and James H-J Yeh

Citation: Appl. Phys. Lett. 75, 1515 (1999);

View online: https://doi.org/10.1063/1.124740

View Table of Contents: http://aip.scitation.org/toc/apl/75/11

Published by the American Institute of Physics

\section{Articles you may be interested in}

Near-field magneto-optics and high density data storage

Applied Physics Letters 61, 142 (1992); 10.1063/1.108198

Near-field optical data storage

Applied Physics Letters 68, 141 (1996); 10.1063/1.116127

Rapid-phase transitions of $\mathrm{GeTe}-\mathrm{Sb}_{2} \mathrm{Te}_{3}$ pseudobinary amorphous thin films for an optical disk memory Journal of Applied Physics 69, 2849 (1991); 10.1063/1.348620

Thermo-optic coefficient of silicon at $1550 \mathrm{~nm}$ and cryogenic temperatures

Applied Physics Letters 101, 041905 (2012); 10.1063/1.4738989

Near-field optical data storage using C-apertures

Applied Physics Letters 97, 073111 (2010); 10.1063/1.3474801

Laser induced crystallization of amorphous $\mathrm{Ge}_{2} \mathrm{Sb}_{2} \mathrm{Te}_{5}$ films

Journal of Applied Physics 89, 3168 (2001); 10.1063/1.1351868

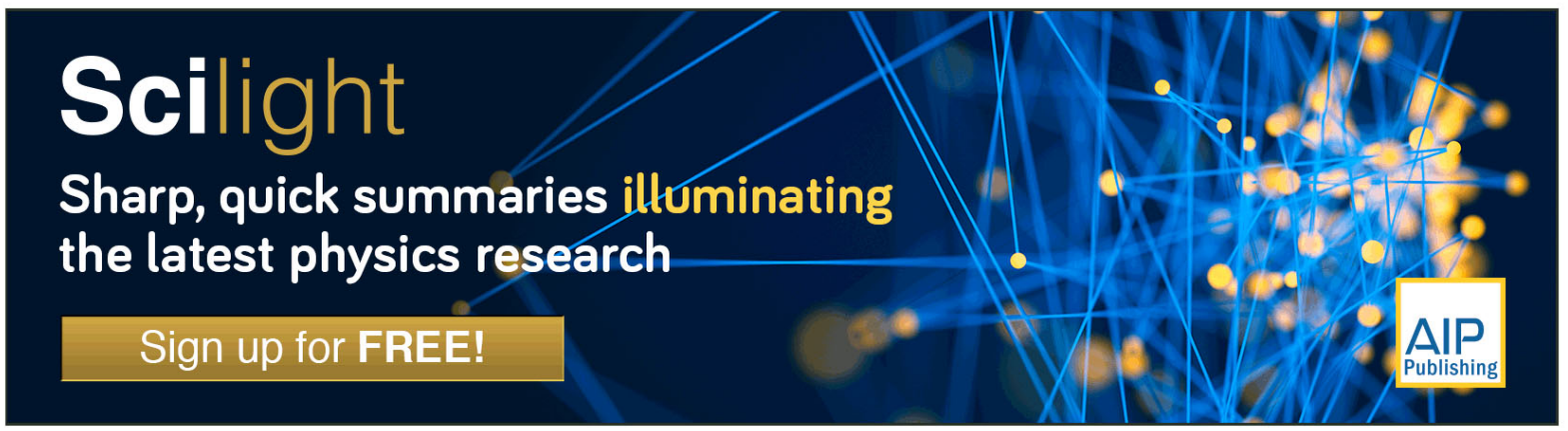




\title{
High-power laser light source for near-field optics and its application to high-density optical data storage
}

\author{
Afshin Partovi, ${ }^{\text {a) }}$ David Peale, ${ }^{\text {b) }}$ Matthias Wuttig, ${ }^{\text {c) }}$ Cherry A. Murray, George Zydzik, \\ Leslie Hopkins, Kirk Baldwin, William S. Hobson, James Wynn, John Lopata, Lisa Dhar, \\ Rob Chichester, and James $\mathrm{H}-\mathrm{J}$ Yeh ${ }^{\mathrm{d})}$ \\ Bell Laboratories, Lucent Technologies, 600 Mountain Avenue, Murray Hill, New Jersey 07974
}

(Received 3 May 1999; accepted for publication 1 July 1999)

\begin{abstract}
A laser light source for high-resolution near-field optics applications with an output power exceeding $1 \mathrm{~mW}\left(10^{4}\right.$ times the power from previous sources) and small (300 nm square to less than $50 \mathrm{~nm}$ square) output beam size is demonstrated. The very-small-aperture laser (VSAL) tremendously expands the range of applications possible with near-field optics and increases the signal-to-noise ratios and data rates obtained in existing applications. As an example, 250-nm-diam marks corresponding to $7.5 \mathrm{~Gb} / \mathrm{in}^{2}{ }^{2}$ storage density have been recorded and read back in reflection and transmission on a rewritable phase-change disk at $24 \mathrm{Mb} / \mathrm{s}$ with a 250-nm-square aperture VSAL. VSALs potentially enable data storage densities of over $500 \mathrm{~Gb} / \mathrm{in}^{2}{ }^{2}$ (up to 100 times today's magnetic or optical storage densities). (C) 1999 American Institute of Physics.
\end{abstract}

[S0003-6951(99)04734-8]

In recent years, techniques such as confocal microscopy, ${ }^{1}$ solid immersion lens (SIL) microscopy, ${ }^{2}$ scanning interferometric apertureless microscope (SIAM), ${ }^{3}$ and near-field scanning optical microscopy (NSOM), ${ }^{4,5}$ have extended the spatial resolution of optical microscopy beyond the diffraction limit of $\sim \lambda / 2$, where $\lambda$ is the wavelength of light. Of these techniques, SIAM and NSOM have demonstrated the highest resolution to date. While SIAM can achieve near-atomic resolution of $1 \mathrm{~nm},{ }^{3}$ it is primarily an instrument for measuring the local susceptibility of a sample with very high spatial resolution. Many important applications in spectroscopy, photolithography, and data storage use the contrast obtained from absorption, reflection, or Kerr and Faraday polarization rotation of light. These applications require the interaction of a sufficiently strong light source with the sample. In NSOM, a sub-wavelength-size light source such as light exiting a small aperture of size $d$ in an opaque screen (typically, metal) is raster scanned in close proximity $(<d / 2)$ of a sample and the light transmitted or reflected from the sample is measured to produce a high-resolution image. Resolution down to $12 \mathrm{~nm}(\lambda / 43)$ has been obtained experimentally. ${ }^{4} \mathrm{NSOM}$ has been used in areas as diverse as biology, surface chemistry, material science, data storage, and characterization of optical communication devices such as lasers, waveguides, and fibers. ${ }^{6}$ Fabrication of a high output power light source has been a critical issue in the progress of NSOM. Tapered, metal-coated optical fibers ${ }^{4}$ provide a dramatic power improvement over earlier probes $\left(10^{5} \times\right.$ quartz tip ${ }^{7}$ and $10^{4} \times$ the pipette $\left.{ }^{8}\right)$ making them the predominant light source for near-field optics in the last de-

\footnotetext{
a)Electronic mail: afp@lucent.com

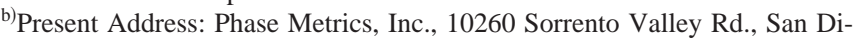
ego, CA 92121

${ }^{c)}$ Present Address: I. Physikalisches Institut der RWTH Aachen, Lehrstuhl für Physik neuer Materialien, 52056 Aachen, Germany.

d) Present Address: TECOM Co., Ltd., 23 R\&D Road 2, Science-Based Industrial Park, Hsin-Chu, Taiwan.
}

cade. Output powers of up to $50 \mathrm{nW}$ out of $100 \mathrm{~nm}$ aperture diameter tapered fibers at $\lambda=514.5 \mathrm{~nm}$ can be obtained for $\sim 10 \mathrm{~mW}$ power input to the fiber. However, most of the power is lost through heat dissipation in the taper region of the fiber where it is operating beyond waveguide cutoff. Further increase of the input power causes the metal coating to melt and catastrophically destroy the light source. Thus, despite intense research in the last decade, the output power from fiber sources has not increased substantially.

Here, we report on a light source consisting of a laser diode with a metal-coated facet where a small aperture has been created. The very-small-aperture laser (VSAL) demonstrates more than a $10^{4} \times$ increase of output power over coated tapered fibers with comparable aperture diameters. This advance opens up the range of applications possible with near-field optics and increases the signal-to-noise ratios and data rates obtained in existing applications. The small size of the VSAL (typical size $750 \mu \mathrm{m} \times 300 \mu \mathrm{m} \times 150 \mu \mathrm{m}$ ) and its ability to generate light as well as to simultaneously detect sample reflectivity changes allows compact NSOM microscopes and other high-resolution optical systems to be designed.

As an example, using a 250-nm-square aperture VSAL at $\lambda=980 \mathrm{~nm}$, we have demonstrated rewritable recording and readback of 250 -nm-diam marks at $7.5 \mathrm{~Gb} /$ in. $^{2}$ which is three times the density of a digital versatile disk read-only memory (DVD-ROM) at a $24 \mathrm{Mb} / \mathrm{s}$ data rate (over $2 \times$ DVDROM). Higher densities can be achieved with the optical near-field aperture storage technique (ONFAST) using smaller-aperture VSALs.

The concept of the VSAL can be applied to diode lasers of any wavelength and design. To fabricate the VSALs, edge-emitting ridge waveguide lasers at $\lambda=980 \mathrm{~nm}$ were used. The lasers used had an output beam size of about 350 $\mathrm{nm}$ full width at half maximum (FWHM) vertically (in the direction of the laser junction). Laterally, the mode size is dependent on the width of the ridge, which is defined by 


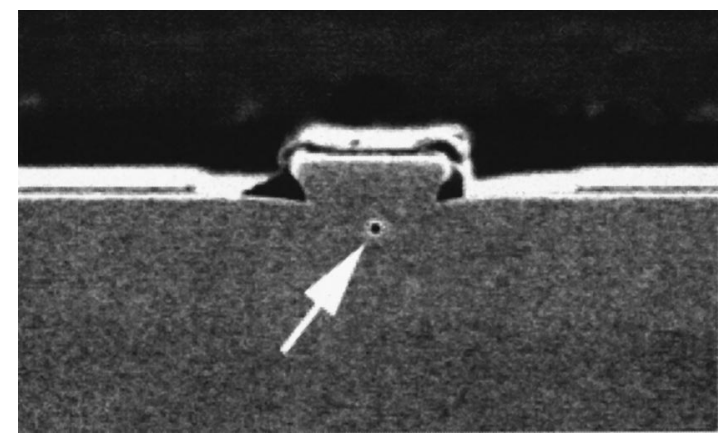

FIG. 1. Focused ion-beam (FIB) image of the metallized front facet of the VSAL. The white arrow shows a 200-nm-square aperture etched into the metal layer by the FIB.

photolithography and etching. Our lasers have typical ridge widths of $2.5 \mu \mathrm{m}$. With a high reflectivity back facet coating and a low reflectivity front facet, the laser threshold is at 21 $\mathrm{mA}$. At $40 \mathrm{~mA}$, an output power of $13 \mathrm{~mW}$ from the front facet is obtained.

After fabrication of the ridge waveguide laser and the back facet coating, a multilayer dielectric coating followed by a multilayer metal coating is deposited on the front facet of the laser. The coating is designed to maximize the power output and readback sensitivity in reflection for the VSAL during operation. Next, an aperture with the desired shape and size is created in the metal coating using a focused ionbeam (FIB) machine that allows imaging as well as etching of samples with a Gallium beam with a $10 \mathrm{~nm}$ resolution. Figure 1 shows the facet of a laser diode with a 200-nmsquare aperture created on the front facet. After processing a laser bar consisting of 25 lasers, individual VSALs are cleaved from the bar. We have fabricated a series of VSALs with apertures in the range of $400-50 \mathrm{~nm}$ square. Figure 2 shows the far-field power from different square aperture size VSALs at $40 \mathrm{~mA}$. Far-field output powers range from $1 \mathrm{~mW}$ for $250-\mathrm{nm}$-square aperture VSALs to $10 \mu \mathrm{W}$ from $50-\mathrm{nm}$ square apertures. However, the power per unit emission area remains around $10 \mathrm{~mW} / \mu \mathrm{m}^{2}$ throughout this aperture size range. The inset in Fig. 2 shows the far-field output power from a 150-nm-square aperture VSAL as a function of the current. This VSAL has a threshold current of $14 \mathrm{~mA}$ and a far-field output power of $0.3 \mathrm{~mW}$ at $40 \mathrm{~mA}$. Typical VSALs can be operated at up to $80 \mathrm{~mA}$ current with proportionally

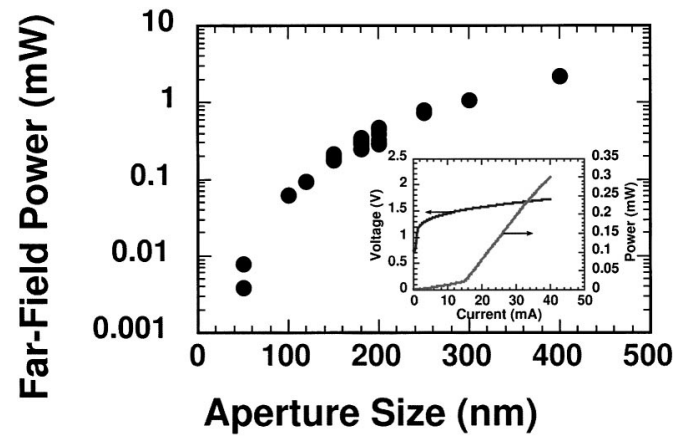

FIG. 2. The far-field power as a function of square aperture size for a series of VSALs at $40 \mathrm{~mA}$ current measured by a collecting lens and a detector with NA $=0.92$. The inset shows the far-field output power from a $150-\mathrm{nm}$ square aperture VSAL. The voltage across the VSAL as a function of the current is also shown.

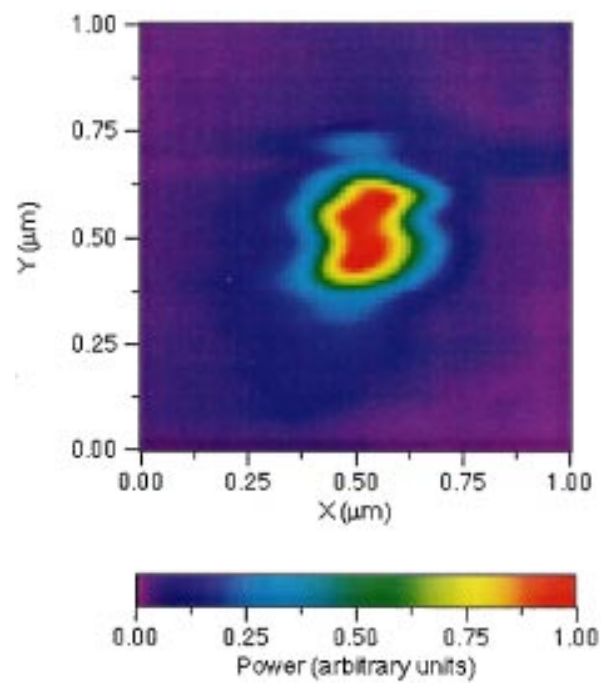

FIG. 3. NSOM image of the output beam from a 50-nm-square aperture VSAL. The resolution of the NSOM image in this case is limited by the 250 nm NSOM fiber aperture diameter used. No light leakage is observed outside the aperture.

larger output powers. It must be remembered that the power measured in the far field from a sub-wavelength-size aperture represents only a small fraction of the near-field power available. ${ }^{5}$ This near-field power is the power that interacts with a sample and can be used to modify its properties in applications such as data storage, spectroscopy, and lithography.

The optical power throughput from an apertured metallic screen is highly dependent on the wavelength of the light and the size of the aperture. ${ }^{7} \mathrm{We}$ can expect that shorterwavelength VSALs such as the $\lambda=650 \mathrm{~nm}$ red lasers or shorter future wavelengths instead of $\lambda=980 \mathrm{~nm}$ used here would provide larger output powers for a given incident laser intensity and aperture size.

The measured far-field powers from VSALs are over $10^{4}$ times the power measured from tapered fibers of similar aperture sizes. One reason for this enhancement is that in a tapered source, much of the power is lost in the waveguide region where the width is reduced below the waveguide cutoff. With an apertured flat screen, this taper loss does not exist. Another reason for the enhancement is that a VSAL is an active source where the photons not transmitted through the aperture can be recycled in the resonator. A similar increase in transmission efficiency in microwave resonant structures has been demonstrated recently. ${ }^{9}$

NSOM imaging has been used in the past to study the emission profile of diode lasers with high resolution. ${ }^{10} \mathrm{We}$ used a tapered, metal-coated fiber to obtain an image of the output of a 50-nm-square aperture VSAL in a NSOM microscope. Figure 3 shows the NSOM image of the output beam. The resolution of the NSOM image in this case is limited by the $250 \mathrm{~nm}$ fiber aperture diameter but shows the high degree of confinement of the output beam from the VSAL.

While the sharp tip of a tapered optical fiber allows the light source to be brought close to a sample, using a flat facet light source for NSOM has been demonstrated previously. ${ }^{11}$ Such techniques can be applied to using VSALs in NSOM. If necessary, small tip size VSALs for NSOM applications can be fabricated using etching or polishing of the VSAL sub- 


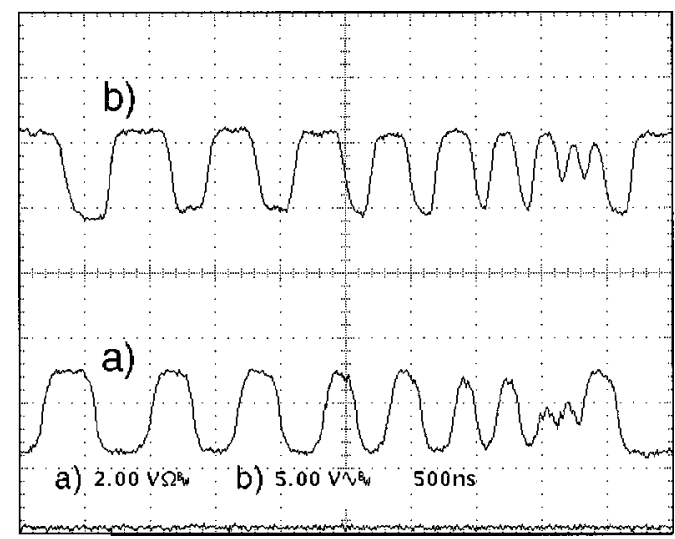

FIG. 4. Transmitted readback signal (a) 2 V/unit for a data pattern with varying written amorphous mark lengths and the reflection readback signal (b) 5 V/unit. The VSAL was operating at $25 \mathrm{~mA}$ and the threshold of this laser was at $15 \mathrm{~mA}$. The media velocity was $4.8 \mathrm{~m} / \mathrm{s}$. The time scale is 500 $\mathrm{ns}$ and the smallest marks reproduced here correspond to the $400 \mathrm{~nm}$ marks at the $18 \mathrm{Mb} / \mathrm{s}$ data rate [using the RLL $(2,7)$ code where each mark represents a $3 / 2$ data bit]. The VSAL reflection signal was obtained by amplifying by $1000 \times$ the ac-coupled component of the VSAL voltage. The reflection signal (before amplification) of $6 \mathrm{mV}$ is $0.4 \%$ of the dc voltage. The transmission signal was obtained by collecting the light transmitted through the disk with a lens $(\mathrm{NA}=0.4)$ and a detector.

strate. However, for many applications, the flatness of the VSAL facet does not pose a problem.

We have applied our VSAL to data storage by mounting a 250-nm-square aperture VSAL to the metallized back face of a magnetic storage-type slider. ${ }^{12,13}$ Accurate mounting was achieved using a reference plane to ensure that the output facet of the mounted VSAL is on the same plane as the air-bearing surface of the slider rails. The slider is in turn attached to a magnetic-type suspension that controls the fly height statically. We fabricated a near-field multilayer phasechange recording disk on a magnetic storage flat glass substrate. The $\mathrm{Ge}_{0.4} \mathrm{Sb}_{0.1} \mathrm{Te}_{0.5}$ recording material used is similar to the material used in digital versatile disk random access memory (DVD-RAM) disks and is rewritable. The disk is designed to have readback contrast in transmission as well as in reflection. To prepare for writing, we crystallized the entire disk by a rapid thermal anneal (RTA) process. To write a desired data pattern, the VSAL is switched to a high output power for the length of a mark to write an amorphized (lowreflectivity, high-transmission state) mark. Erasure is achieved through recrystallization with a lower laser power to a high-reflectivity, low-transmission state.

Recently, the optical gain of a fiber laser probe has been used to obtain large NSOM signals in reflection. ${ }^{14}$ Use of the reflectivity modulation for readback of optical disks with a laser diode in the far field ${ }^{15}$ and near field ${ }^{16,17}$ has also been previously demonstrated. Operating near laser threshold, the reflection readback signal can be measured by monitoring the output power from the back facet. ${ }^{16,17}$ The reflection signal can also be obtained more easily by measuring the voltage across the laser diode resulting from modulation of the laser impedance. ${ }^{15}$ ONFAST readback in reflection can be achieved by operating the VSAL near laser threshold and detecting the voltage modulation as the amorphized (low reflectivity) and crystalline (high reflectivity) marks pass in front of the aperture.

Flying a 250-nm-square aperture VSAL with a VSAL facet/recording layer spacing of $75 \mathrm{~nm}$, data tracks were written by switching the VSAL between 0 and $55 \mathrm{~mA}$ to write amorphized marks. Figure 4 shows the reflection (a) and transmission (b) readback signal from written amorphized marks of varying lengths. Large, low-noise signals were obtained in reflection as well as transmission. The shortest period patterns in this image correspond to $400 \mathrm{~nm}$ marks and $400 \mathrm{~nm}$ spaces and were written and read back at an $18 \mathrm{Mb} / \mathrm{s}$ data rate. A repeated pattern of $250 \mathrm{~nm}$ marks and $250 \mathrm{~nm}$ spaces was also written and read back at 24 $\mathrm{Mb} / \mathrm{s}$ showing the subwavelength resolution possible with VSALs. The reflection (VSAL voltage modulation) and transmission signals had a $45 \mathrm{~dB}$ carrier-to-noise ratio (CNR) with a $30 \mathrm{kHz}$ measurement bandwidth for the 250 $\mathrm{nm}$ pattern. Such a CNR is sufficient for data storage applications. The width of the data track was $250 \mathrm{~nm}$ and adjacent tracks could be written at $500 \mathrm{~nm}$ spacing. This demonstration corresponds to $7.5 \mathrm{~Gb} / \mathrm{in}^{2}{ }^{2}$ storage density with a $(2,7)$ run-length limited (RLL) coding scheme. This recording density $(3 \times$ DVD-ROM) demonstrates the high densities achievable with ONFAST. Using smaller-aperture VSALs and flying closer to the media, much higher densities should be possible.

The smallest beam size and resolution achievable from a VSAL source as well as any other apertured near-field source is given by the penetration depth of the electromagnetic field into the screening metal. This penetration depth limits the beam size to around $30 \mathrm{~nm}$ FWHM. ${ }^{18}$ Assuming that sufficient power from the VSAL is available for writing with these small diameter beams, densities of $\sim 540 \mathrm{~Gb} / \mathrm{in}^{2}{ }^{2}$ (i.e., $100 \times$ today's magnetic storage) may be achievable.

${ }^{1}$ T. Wilson and C. Sheppard, Theory and Practice of Scanning Optical Microscopy (Academic, London, 1984).

${ }^{2}$ S. M. Mansfield and G. S. Kino, Appl. Phys. Lett. 57, 2615 (1990).

${ }^{3}$ F. Zenhausern, Y. Martin, and H. K. Wickramasinghe, Science 269, 1083 (1995).

${ }^{4}$ E. Betzig, J. K. Trautman, T. D. Harris, J. S. Weiner, and R. L. Kostelak, Science 251, 1468 (1991).

${ }^{5}$ D. W. Pohl, W. Denk, and M. Lanz, Appl. Phys. Lett. 44, 651 (1984).

${ }^{6}$ M. A. Paesler and P. J. Moyer, Near-Field Optics (Wiley, New York, 1996).

${ }^{7}$ U. Dürig, D. W. Pohl, and F. Rohner, J. Appl. Phys. 59, 3318 (1986).

${ }^{8}$ A. Harootunian, E. Betzig, M. Isaacson, and A. Lewis, Appl. Phys. Lett. 49, 674 (1986).

${ }^{9}$ R. D. Grober, R. J. Schoelkopf, and D. E. Prober, Appl. Phys. Lett. 70, 1354 (1997).

${ }^{10}$ S. K. Buratto, J. W. P. Hsu, J. K. Trautman, E. Betzig, R. B. Bylsma, C. C. Bahr, and M. J. Cardillo, J. Appl. Phys. 76, 7720 (1994).

${ }^{11}$ U. Ch. Fischer, U. T. Dürig, and D. W. Pohl, Appl. Phys. Lett. 52, 249 (1988).

${ }^{12}$ A. Partovi, D. Peale, C. A. Murray, G. Zydzik, L. Hopkins, J. H-J Yeh, M. Wuttig, R. Chichester, L. Dhar, D. Vakhshoori, W. S. Hobson, J. Wynn, and J. Lopata, "Ultra High Density Near-Field Optical Storage," in Conference on Lasers and Electro-optics (CLEO), Vol. 9, 1996, OSA Technical Digest Series (Optical Society of America, Washington, DC, 1996), pp. 195-196.

${ }^{13}$ A. Partovi, " Optical Near-Field Aperture Storage Technique (ONFAST) for High Density, High Performance Data Storage Applications,' 'in Joint International Symposium on Optical Memory and Optical Data Storage 1999, SPIE, (1999), Vol. 3864, pp. 352-354.

${ }^{14}$ E. Betzig, S. G. Grubb, R. J. Chichester, D. J. DiGiovanni, and J. S. Weiner, Appl. Phys. Lett. 63, 3550 (1993).

${ }^{15}$ Y. Mitsuhashi, J. Shimada, and S. Mitsutsuka, IEEE J. Quantum Electron. 17, 1216 (1981).

${ }^{16}$ Y. Katagiri and H. Ukita, Jpn. J. Appl. Phys., Suppl. 28-3, 177 (1989).

${ }^{17}$ K. Goto, T. Sato, and S. Mita, Jpn. J. Appl. Phys., Part 1 32, 5459 (1993).

${ }^{18}$ E. Betzig and J. K. Trautman, Science 257, 189 (1992). 